\title{
Dismenorrea orgánica extra uterina
}

(Trabajo presentado el 14 de junio de 1951)

\section{Por el doctor A. ESPINOSA ESPINOSA}

Con sumo placer presento esta charla que en asocio de distingnidos colegas del ICSS, leímos ante la décima reunión científica de dicho Instituto.

En ella y más que todo para facilided didéctica y comodidad de exposición para los varios ponentes nombrados por la dirección cientifica, resolvimos no entrar ell discusión. sobre la denominación de la entidad que nos ocupa y aceptamos la de dismenorrea. así como también la dividimos en orgánica intrauterina, orgánica extrauterina, funcional y psicogena, encargándose el internista $y$ el farmacólogo de la introduceión y el punto de vista terapéution sintomático, respectivamente.

Conocidas son de todos vosotros las denominaciones que se han propuesto a ssta entidad de la patología, pareciéndome la más logicea y aceptable la de algomenorrea, ya que conocéis que el dolor es el síntoma capital en el tema que nos ocupa, ya sea del tipo expuisiro, c. congestivo, quedando relegados a segundo término los otros síntomas que la acompañan. Por otra parte el térırino dismenorrea etimológicamente hahlando, indiea dificultad, alteración. trastormo de la menstruación que en la mayoría de los casos no se comprueba como dependiente exclusivamenti. del mecanismo menstrual.

El término algomenorrea, indiea solamente el sintoma capital y no prejuzga sobre su etiología. Soy partidario (i) universalizar la terminología médi(a y por ello os traigo mi opinión sobre este punto para que lo discutamos ampliamente.

No entro en detalles de clasificarión. pero acá también creo lógico y científico clasificar la algomenorrea. en primitiva y seeundaria, no trmiendo en rrenta el factor tiempo de aparición, sino la etiología, agregándole después (1. su estudio el segundo apelativo, v. g. : algomenorrea secundaria inflamatoria, Ja cual es una de las afecciones yur hioy vamos a considerar.

De acuerdo con lo dicho, el tema de hoy comprenderá una parte de las algomenorreas secundarias que para fa- -ilitar la descripción divido en: inflamatorias, tumorales, venosas, nerviosas, intestinales y urinarias. En su mayoría presentan el tipo doloroso congestivo. 
Inflamatorias. No escapa a llatie la importancid de este tipo de algomenorrea, siendo bien ronocidas de todos sus *aracteristicas rlinicas $y$ andiomopatoJigicas.

Dentro de las formas elimicas presentan un mayor interés las formas eroni“as y subagndas ya estén localizadas in las trompas, ovarios, paramotrios o solamente en el peritoneo pélvioo. romo secuela de los procesos inflamatrios do estos órganos.

La algomenorrea de este tip. es debida a la hipereangestión uterina con distención del perimetrio y de los procesos adherenciales y por lo tanto, también a las contracciones uterinas.

Paso por alto por ser de todos concecida su etiología y patogenia y sólo hago resaltar la facilidad de que pase inalverticlo en el puerperio, un proceso in. flamaiorio tubario que producirá como conservencia tardía la algomenorrea y esterilidad secunciarias.

El iratamiento de estas formas clínicas se hace a base de estrógenos, diatermia, antibióticos y persuflaciones. Estas b́ttimas indicadas más para conocer el resultado del tratamiento o bien romo medio adymante para tratar mun obstruceicin tubaria.

Aproverho la oportmidad para hater notar que los procesos agudos mal curados. es decir, clínicamente tratalos como hasta hace poco tiempo lo hairia. mos, e(c)introlando solamente la parte rlíniea y hematológica, para afirmar o infirmar la curación de un proceso inflamatorio tubario, presenta una enorme falla, ya que así no conocemos si n órgano puedó funcionalmente norma!

C'ono ronsecmencia nos arriesgrmos a que posteriormente estas enfermas, ge- seralusente en edat aetwa gental, pre senten una obstruición ron pequerios foeos inflamatorios yue serán causa de algomenorrat semondaria y esterilidad.

Para llegar a la afirmarión drimitiva de coración es necesario a mi noodo de ver, la presuflación tubaria.

Volviendo al tema de] tratamiento de] proeseso inflamatorio y ante of praraso de los reenrsos médieos se debe ir a luma rirugia tan eomservadora como las lesiones lo permitan, aprovechando para harer eomo medida suplementaria y de gran importancia la resección del pre-sacro (Cotte) y correguir cualquier otra anormalidad en la pelvis, halladia o no en los exámenes clínicos.

Da paso anoto que la operación de Cotte, usadia en la algomenorrea grave. sin lesión aparente, tiene 10 fracaso que oscila entre el 2 y el $20 \%$, según diver sas estaclisticas, motivo por el cual hoy se usan la prueba de los estrógenos o el bloyneo simpático, para deterninar hasta donde es posible, si su cansa es psicógena o no.

Anoto solamente que el bloqueo simlático lumbar, da resultados idéuticos a la operación de Cotte, pero de cará. ter temporal y en tal raso, indicari la •irugía. Sera negativa en las dismenomeas puirégernas.

Tumoreles. En lo referente a tumures. ocupa lugar de preferenela la endonietrosis erterno, que por su aceión irritativa dr distención $y$ ann hemorrácriat peritomeal. lleva a la algomenorrea segúm estadistivas extranjeras se halla en el 20 al 30\%; de la eirugía ginecológica ved sll importancia!

Comoritas son de torlos, las diveras loralizariomes: ovárica, tubárica, tabiyme repto-vaginal. vagino-vesiral, fondo de 
Saro de Douglas, ligamentos e intestinos amén de otras formas extraperitomeales, bastante raras.

El diagnostico bastante dificil sie ha re al comprobar lesiones tumorales en Jos diversos sitios anotados, acompañados de algomenorrea secundaria, tipo congestive, que generalmente va en aumente yen parentes eon caremeia ab. soluta de antecerentes infeceiosos.

Es de gran ayuda en el diagnésice el uso de hiperhemiantes y vasodilatadores, como los estrógenos y la diatermia, pues con ellos si existe la endometrosis deben aumentar los dolores premenstruales. Lo contrario sucede en los procesos inflamatorios.

El tratamiento puede ser hormonal, radioterápice o quirírgico. Su seleceión st hace segín la edad de la paciente, el grado de las lesiones, su evolueión e intensidad relinicas.

En general debe darse preferencia al ratamiento quirúrerico que da mayores sarantías desde todo punto de vista, a la vez que permitirá practicar el traamiento más conveniente a cada caso.

fin embargo, existen casos muy avanaados en donde usaremos la radioterapia “astración para (*ontrolar el aumento de la lesión y por ende de la dismenorred. Habrá casos en los cuales e] lise de la testorana, enidando de no llesall a la dosis virilizante, se harí necesaria como medida paliativa y mientras se corrigen las lesiomes que impidan ir $\therefore$ la cirugía inmediata. Nunca como tratamiento (ontimuo y definitivo.

Do las demás tumorariones pélvicas "xtranterinas, solo resta agregar que senerahnonte se ponen de presente por -I1 manifestaleiones dolorosas de rem?ursion a los filetes nerviosos a verinos a los órganos adyacentes y que rara rez producen algomenorrea.

Algunas formas clínicas como la ptosis ovárioa en el Douglas o la ovaritis eselero-equistica, podrian incluírse affuí, pro la falta de constancia en la producrión del dolor menstrual en estas easos, halee que sólo las meneione do paso.

Mereece citarse en este punto el he. matorele pelviano crónico, que aumenando no es un verdadero tumor, al enrapsularse forma un falso quiste que $\therefore$ produce algomenorrea, por idéntico mecanismo al de las lesiones inflamatorias. Su tratamiento es igual.

Timosas. El varicocele pelviano de finto ange en otro tiempo, a mi modo d. ver, ha peraido gran cantidad de adeptos y si logramos individualizarlo (omo entidad nosológica pura, bien poGría ser rausa de algomenorrea. Sin tratamiento es quirúrgico.

Niriosos. Algunos han invorado romo eausa de algomenorrea, lesiones nerriosas de los plejos hipogástricos, debido a hallazgos anatomo-patológicos de neuritis.

Sil importancia es urande, eonceida la taxiologia simpático-parasimpático y rebirla probablemente a regímenes dietéticos rarentes en vitaminas, en esperjal do Ja vitamina $B_{1}$. De ahí la jumpertancia de una dieta bien balanceada, rica en vitaminas, proteinas $y$ de una torapéntica vitaminoterápica especialmente en enfermas desnutridas y de tipo asténico-hipoplásico. Serían ias bilias de este grupe que no presentan holo? de tipo congestivo.

Trimarias. Algunas Jesiones urinarias pueden presentar este tipo de algome- 
serma, en especial las inflamaciones crinicas de la rejiga y la acodadura wreteral inferior. Por eso, en ciertos casos, sospecho de presentar tales lesiones. debemos pedir el concepto del urólogo. quien hará, según el caso, la citoscopia, el cateterismo ureteral y la uretoropirlografía ". instituirá el trata1:ientu adecuain.

Intestinales. Finalmente lesiones rónicas inflamatorias del apéndice, esperialmente en su localización pélvica y como consecuencia de procesos periajendiculares, a continuación de una sulpingo-ovaritis o no, han sido dadas

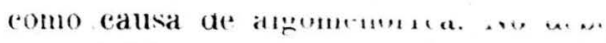
mos olvidar ello, ya que en pocos casos publicados la apendicectomía ha dado lai cura definitiva.

Tampoco debemos olvidar las inflamaciones crónicas del sigmoide, y en especial la sigmoiditis amibiana. En su suspecha, estos casos debemos remitiplon al gastroenterólogo, para sll estudio, después de descartar todas la lesiones, Ya estudiadas.

Su patogenia muy discutida, puede ser debida al dolor hipogástrico que viene a intensificar una algomenorrea psicógena moderada, que hasta entonces pasaba desapercibida para la enferma. 